\title{
The therapeutic efficnecy of ranolazine in comparison with conventional therapy in diabetic individuals with ischemic heart disease; a randomized clinical trial
}

\author{
Bahram Fariborz Farsad $^{\oplus}$, Seyed Mostafa Alavi ${ }^{{ }^{*} \oplus}$, Golshid Ghorbanian², Fanak Fahimi ${ }^{2}$, Zahra \\ Ghaemmaghami $^{1}$, Ali Zahed Mehr ${ }^{3}$, Hooman Bakhshandeh ${ }^{1}$ \\ ${ }^{1}$ Rajaie Cardiovascular Medical and Research Center, Iran University of Medical Sciences, Tehran, Iran \\ ${ }^{2}$ Clinical Pharmacy Department, School of Pharmacy, Shahid Beheshti University of Medical Sciences, Tehran, Iran \\ ${ }^{3}$ Cardiovascular Intervention Research Center, Rajaie Cardiovascular Medical \& Research Center, Iran University of Medical Sciences, \\ Tehran, Iran
}

\section{A R T I C L E I N F O}

Article Type:

Original

\section{Article History:}

Received: 9 June 2019

Accepted: 23 August 2019

Published online: 9 September 2019

\section{Keywords:}

Glycated hemoglobin A

Heart diseases

Hypoglycemic agents

Nitroglycerin

Ranolazine

\begin{abstract}
A B S T R A C T
Introduction: Patients with diabetes mellitus (DM) have higher risk of coronary artery disease (CAD) and angina than the general population.

Objectives: We sought to assess the impact of ranolazine as an antianginal agent to diminish hemoglobin A1c ( $\mathrm{Hb}$ Alc) level in diabetic individuals under treatment with metformin and gliclazide.

Patients and Methods: This study was a randomized parallel-group clinical trial to compare the therapeutic effects of ranolazine versus conventional treatment in patients with concomitant T2DM and stable angina. TERISA (type 2 diabetes mellitus evaluation of ranolazine in subjects with chronic stable angina) was employed to assess the efficiency of ranolazine in lowering the frequency of angina attacks. The study was performed on diabetic patients with symptomatic angina under treatment with either sublingual nitroglycerin or up to two types of antianginal agents. The patients in the intervention group received $1000 \mathrm{mg}$ ranolazine twice a day while patients in the control group were administrated with conventional treatment. Reduction in baseline Hb Alc level was determined at 4 and 8 weeks after the interventions.

Results: Ranolazine therapy resulted in up to $15 \%$ absolute reduction in $\mathrm{Hb}$ Alc level in diabetic patients who had baseline HbAlc level $>7 \%$. Ranolazine was more efficient in reducing $\mathrm{Hb} \mathrm{Alc}$ in patients with poorly controlled disease (i.e. HbA1c $>8 \%, P=0.017$ ). Ranolazine did not affect the glycemic status of patients concomitantly treated with antidiabetic therapy $(P=0.560)$.

Conclusion: Concurrent administration of ranolazine along with anti-diabetic treatments (i.e. metformin and gliclazide) was safe to reduce the level of HbAlc in patients with poorly controlled diabetes and stable angina.

Trial Registration: The protocol was approved in Iranian Registry of Clinical Trials under the number of IRCT20161026030511N3; https://www.irct.ir/trial/24223, Ethical code; R.SBMU. PHNM.1394.308.
\end{abstract}

Implication for health policy/practice/research/medical education:

Ranolazine can improve quality of life and exercise tolerance and reduce angina frequency. Ranolazine can also improve HbA1c level in individuals with concomitant type 2 diabetes mellitus and chronic stable angina without imposing any adverse effects on kidneys.

Please cite this paper as: Fariborz Farsad B, Alavi SM, Ghorbanian G, Fahimi F, Ghaemmaghami Z, Zahed Mehr A, Bakhshandeh $\mathrm{H}$. The therapeutic efficnecy of ranolazine in comparison with conventional therapy in diabetic individuals with ischemic heart disease; a randomized clinical trial. J Renal Inj Prev. 2020; 9(1): e04. DOI: 10.15171/jrip.2020.04.

\section{Introduction}

Type 2 diabetes (T2DM) is a global health problem particularly in developed countries. Diabetes mellitus (DM) increases the risk of coronary artery disease (CAD) and angina $(1,2)$ and is a significant predictor of mortality and comorbidity in patients with cardiovascular diseases and angina $(3,4)$. Hyperglycemia may accelerate the risk of atherosclerosis, a chronic inflammatory process which is known as the main contributor to cardiac complications. According to these, it is highly desirable to develop drugs to concurrently treat both T2DM and CAD.

Ranolazine is the first line antianginal drug with cardioprotective effects and no impacts on cardiac function, heart rate or blood pressure (5). Ranolazine 
decreased the need for administration of sublingual nitroglycerin and the frequency of angina $(6,7)$ and also improved glycemic control and quality of life in diabetic patients (8). Ranolazine is believed to reduce myocardial ischemia and angina incidence through inhibiting cardiac slow sodium currents and reducing intracellular sodium and calcium overload as the main contributor to myocardial ischemia (9). The exact mechanism of action of ranolazine to modulate $\mathrm{HbA1c}$ level is still unknown. Ranolazine can regulate late sodium currents channels which are increased in hyperglycemia and promote glucose-stimulated insulin secretion (10-12). HbAlc is a clinically important biomarker for screening long-term glycemic control $(13,14)$.

\section{Objectives}

Since the HbA1c-lowering effects of ranolazine have been reported in previous studies $(3,15)$, clinical trials on this issue are rare. In the current clinical trial, we intended to assess the impacts of ranolazine on HbAlc level and clinical outcomes in patients with DM and stable angina. Our diabetic patients with stable angina were categorized into either intervention (i.e. ranolazine treatment) or control (i.e. conventional treatment) group. All the patients continued to receive conventional anti-diabetic treatments (i.e. metformin and gliclazide). The outcomes included angina frequency and glycemic level.

\section{Patients and Methods}

Study design

This was a parallel-group randomized clinical trial comparing the effects of ranolazine versus conventional therapy in patients with concurrent T2DM and stable angina. The patients who had been diagnosed with these conditions for at least 10 weeks were initially observed for 2 weeks to monitor their health status. Then nitroglycerin was replaced with ranolazine in the intervention group for 8 weeks.

Overall, 60 patients were recruited and divided into either the intervention or control group. The patients were recruited from the diabetes clinic of the Shahid Rajaie Heart Center from June 2015 to January 2016. Patients fulfilling inclusion criteria were randomly selected and allocated into intervention and control groups using the balanced block randomization method.

All the patients were asked to sign an informed consent form. Patients who developed any complication during the study were excluded. After entering the study, the patients were monitored for two weeks to assess their health condition (i.e. phase 1). During this period, the patients received their routine diabetic care (i.e. $500 \mathrm{mg}$ metformin three times a day and $80 \mathrm{mg}$ gliclazide twice a day). They also received $2.6 \mathrm{mg}$ nitroglycerin daily to treat stable angina.

History of daily chest pain or angina and using sublingual nitroglycerin was determined by questionnaire. The patients who received more than two types of antianginal medications had to spend a 2-week wash-out period before entering the study. No patients had been administrated with more than two antianginal drugs or verapamil and/or diltiazem therapeutic diet. The patients who had been treated with either verapamil or diltiazem underwent dosage adjustment by administration of $500 \mathrm{mg}$ maintenance dose of ranolazine twice a day. All the subjects were under observation for two weeks to examine their health status. After 2 weeks of observation, nitroglycerin was replaced with ranolazine in the intervention group while routine treatment continued in the control group.

\section{Study population}

Overall, 60 patients including 29 men and 31 women with mean age of $64 \pm 5$ years old were enrolled in this study. Based on standardized difference (difference/SD) of 0.75 , power of $80 \%$ and an alpha value of 0.05 , the sample size was calculated as 60 ( $n=30$ per each group) using $\mathrm{G}$-power software.

\section{Inclusion criteria}

Diabetic patients with $\mathrm{HbA} 1 \mathrm{c}>6.5 \%$, age $>50$ years old, and chronic stable angina (i.e. persisted angina for more than three months, triggered by physical efforts, and relieved by rest and/or sublingual nitroglycerin) were included. Other inclusion criteria were angiographic evidence of $\geq 50 \%$ stenosis, history of myocardial infarction (MI), CAD diagnosed by cardiac imaging or exercise test, and more than two weeks of treatment with at least two types of antianginal drugs. Patients should have had at least one angina attack per week to enter the study. Patients with T2DM treated with metformin and gliclazide who had systolic blood pressure of 120-160 mm $\mathrm{Hg}$ and presented with dyspnea were also included.

\section{Exclusion criteria}

New York heart association (NYHA) class III and IV patients were excluded from the study (16). Furthermore, patients with the history of acute coronary syndrome during the past two months, those planned for coronary revascularization, and patients who had history of cardiac infraction or transient ischemic attack within the past six months were excluded. Other exclusion criteria were as QTc of less than $500 \mathrm{~ms}$, diastolic blood pressure of more than $110 \mathrm{~mm} \mathrm{Hg}$, and histories of liver cirrhosis, previous treatment with ranolazine, hypersensitivity or intolerance to ranolazine, as well as participation in experiments other than the current study. In addition, patients who were under treatment with trimetazidine, ivabradine or nicorandil, patients undergoing dialysis, and those, who received more than two types of antianginal medications were excluded. 
Exercise test and laboratory investigations to determine $\mathrm{HbA1c}$, fasting blood sugar (FBS) and 2-hours postprandial glucose (2HPP), serum creatinine and lipid profile were performed at baseline, as well as at 4 and 8 weeks after starting the study. Complete clinical data including histories of hypertension, received medications (e.g. antihypertensive drugs such as angiotensin-converting enzyme (ACE) inhibitors and ARBs (angiotensin-receptor blockers), anti-diabetic drugs, calcium channel blockers (CCBs), beta-blockers and nitrates, anti-platelet agents, and antianginal drugs), smoking status, history of MI or coronary artery bypass graft (CABG), and the number of angina episodes were recorded using a standard questionnaire.

The number of angina attacks was recorded during a 24-hour period (i.e. from the evening of one day to the evening of the next day). The number of consumed sublingual nitroglycerin pills per day was also obtained using a self-reported questionnaire. After randomization, the patients in the intervention group were administrated with $500 \mathrm{mg}$ ranolazine for 1 week (i.e. loading dose) which was increased to $1000 \mathrm{mg}$ twice a day (i.e. maintenance dose). In case of tolerance, the maintenance dose was administrated for seven weeks. In the control group, the patients continued their conventional therapy (i.e. $2.6 \mathrm{mg}$ nitroglycerin for angina and metformin and gliclazide for diabetes).

\section{Efficacy assessments}

The primary endpoint of this study was the reduction of $\mathrm{HbA1c}$ in diabetic patients who received either ranolazine or conventional therapy after 8 weeks of the study. The other outcomes were improved treadmill exercise time, reduction in the number of administrated sublingual nitroglycerin pills, and finally reduction in angina episodes per week.

\section{Safety analysis}

Adverse events were self-reported by patients using preprepared forms.

\section{Laboratory measurements}

Blood samples were withdrawn after overnight fasting in the laboratory of the Shahid Rajaie Heart Center. Plasma FBS and 2HPP levels were determined by the plasma glucose oxidase method. Plasma HbAlc was measured by high-pressure liquid chromatography (Hitachi 911 automatic analyzer). Figure 1 shows the CONSORT

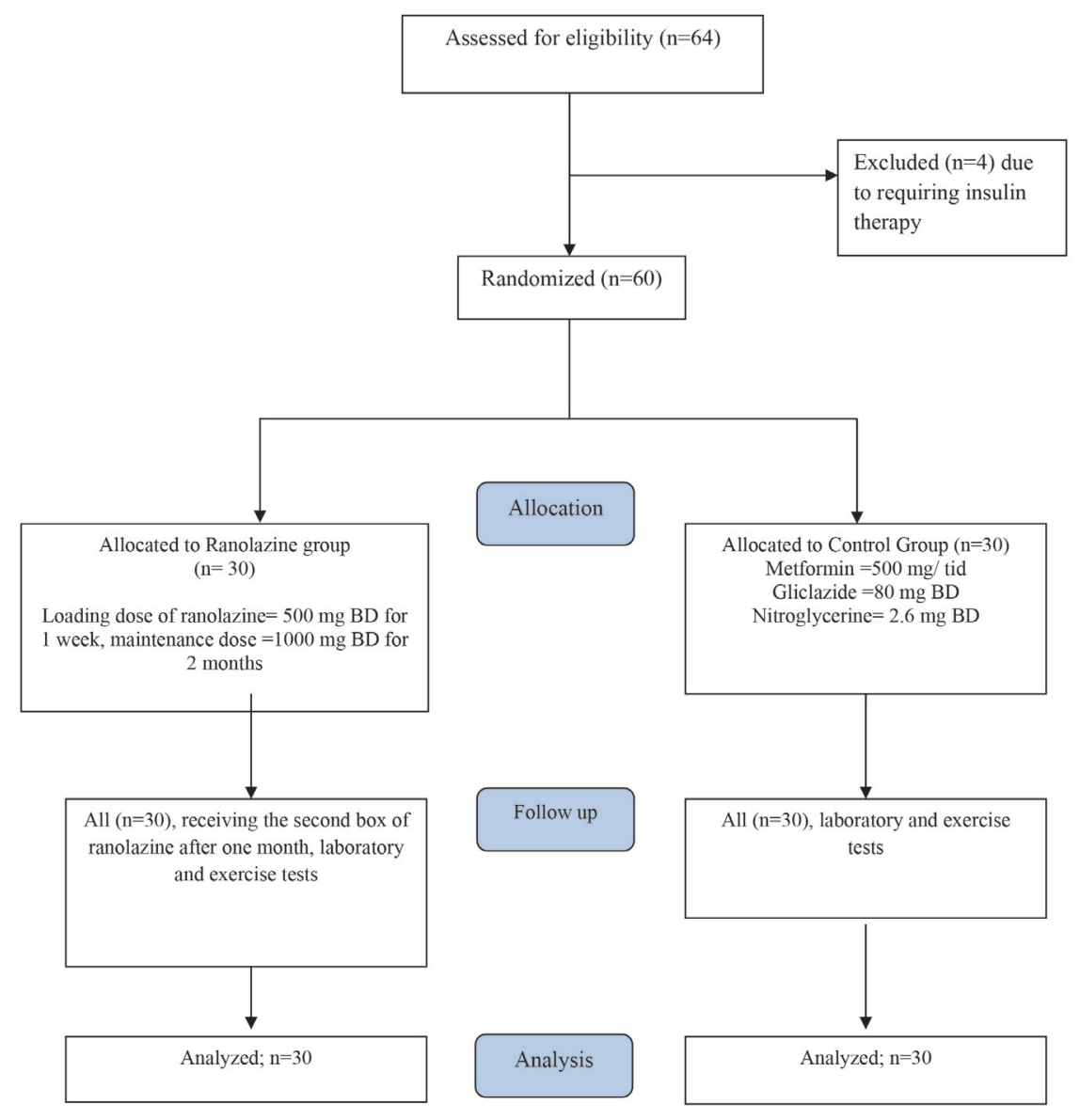

Figure 1. The CONSORT diagram of the study. 
diagram of the study.

\section{Ethical issues}

The research followed the tenets of the Declaration of Helsinki. All study protocols were approved by the institutional ethical committee at Shahid-Rajaei Heart center and Iran University of Medical Sciences. The study was also registered in the Iranian Registry of Clinical Trials (identifier: IRCT20161026030511N3, https://www.irct. ir/trial/24223; Ethical code; R.SBMU.PHNM.1394.308). Written informed consent was obtained from all the participants before any intervention.

\section{Statistical analysis}

Statistical analyses were performed using SPSS software for Windows version 23.0 (SPSS Inc., II, USA). Descriptive statistics were used to present the data as mean \pm standard division (SD) and frequencies. Kolmogorov-Smirnov test was used to assess normal distribution of the variables. Independent samples student $t$ test was used as the parametric test for normally distributed data, and MannWhitney $U$ test was applied as non-parametric test for non-normally distributed variables. Chi-square test was used to compare qualitative variables. The significance level was considered as $P$ value $<0.05$.

\section{Results}

The baseline and clinical characteristics of the studied patients have been shown in Tables 1 and 2 respectively.

\section{Efficacy}

The changes in the levels of HbAlc, FBS and 2HPP in both groups during the study described in Figures 2-5.

\section{Effecs of ranolazine on serum creatinine levels in diabetic patients}

Ranolazine (1000 mg twice a day) did not affect creatinine level during 8 weeks post-treatment (Figure 6, $P=0.182$ ).

\section{Adverse events}

No serious adverse effects and no mortalities were noted during the study in neither of the groups. Mild adverse events have been reported in Table 3 .

\section{Discussion}

For the first time, we here compared the therapeutic effects of ranolazine and conventional treatment in Iranian diabetic patients who suffered from stable angina. There were no significant differences comparing baseline clinical and demographic variables including gender, age, history of MI, history of heart surgery, smoking frequency, and duration of DM between the ranolazine and control groups indicating balanced distribution of these parameters. The primary therapeutic goals in diabetic patients are to maintain the HbAlc level below $7 \%$ and to reduce angina frequency. Our results revealed
Table 1. Baseline characteristics of patients

\begin{tabular}{lccc}
\hline & $\begin{array}{c}\text { Control group } \\
\mathbf{n}=\mathbf{3 0}\end{array}$ & $\begin{array}{c}\text { Ranolazine } \\
\text { group, } \mathbf{n = 3 0}\end{array}$ & P value \\
\hline Male & 15 & 14 & 0.529 \\
Female & 15 & 16 & 0.529 \\
Age (y) & $64(60.75-67.25)$ & $65(58-70)$ & 0.058 \\
History of MI & $46.7 \%$ & $43.5 \%$ & 0.817 \\
History of heart surgery & $52.3 \%$ & $78.3 \%$ & 0.061 \\
Years of smoking & 7.5 years & 7 years & 0.767 \\
Number of smokes per day & 20 & 12 & 0.197 \\
Duration of DM $(y)$ & 8.5 & 6 & 0.382 \\
\hline Abbreviations: $M l$ myocardial infarction; DM, diabetes mellitus.
\end{tabular}

Abbreviations: MI, myocardial infarction; DM, diabetes mellitus.

Table 2. Clinical characteristics of the recruited patients following the start of the treatments

\begin{tabular}{|c|c|c|c|}
\hline & $\begin{array}{c}\text { Ranolazine } \\
\text { group }\end{array}$ & $\begin{array}{l}\text { Control } \\
\text { group }\end{array}$ & $P$ value \\
\hline HbA1c baseline (\%) & $8.3 \pm 1.47$ & $7.85 \pm 0.97$ & 0.035 \\
\hline First month HbA1c (\%) & $7.4 \pm 1.15$ & $7.7 \pm 0.96$ & 0.85 \\
\hline Second month HbA1c (\%) & $7 \pm 0.96$ & $7.8 \pm 0.96$ & 0.072 \\
\hline FBS baseline (mg/dL) & $161 \pm 51.59$ & $128 \pm 54.81$ & 0.002 \\
\hline FBS First month (mg/dL) & $145 \pm 35.69$ & $134.5 \pm 36.1$ & 0.092 \\
\hline FBS Second month (mg/dL) & $131 \pm 33.77$ & $130.5 \pm 26.12$ & 0.467 \\
\hline 2HPP baseline (mg/dL) & $291 \pm 80.56$ & $199.5 \pm 58.94$ & 0.011 \\
\hline 2HPP First month (mg/dL) & $250 \pm 59.25$ & $195.5 \pm 44.65$ & 0.011 \\
\hline 2HPP Second month (mg/dL) & $196 \pm 44.42$ & $192.5 \pm 38.37$ & 0.560 \\
\hline ET baseline & $3.69 \pm 2.97$ & $5 \pm 2.69$ & 0.689 \\
\hline ET First month & $5 \pm 3.52$ & $5.2 \pm 2.97$ & 0.613 \\
\hline ET Second month & $5.5 \pm 3.32$ & $4.65 \pm 2.79$ & 0.353 \\
\hline Angina frequency $w_{1}$ & 7 & 6 & 0.251 \\
\hline Angina frequency $w_{2}$ & 6 & 6 & 0.585 \\
\hline Angina frequency $w_{3}$ & 5 & 6 & 0.046 \\
\hline Angina frequency $\mathrm{w}_{4}$ & 5 & 6 & 0.001 \\
\hline Angina frequency $w_{5}$ & 4 & 6 & 0.003 \\
\hline Angina frequency $w_{6}$ & 4 & 6 & $0.001 \leq$ \\
\hline Angina frequency $w_{7}$ & 4 & 5 & 0.001 \\
\hline Angina frequency $w_{8}$ & 3 & 5 & 0.001 \\
\hline Serum creatinine & $1.024 \pm 0.214$ & $0.974 \pm 0.174$ & 0.399 \\
\hline Serum creatinine $w_{4}$ & $0.986 \pm 0.156$ & $0.996 \pm 0.158$ & 0.835 \\
\hline Serum creatinine $\mathrm{w}_{8}$ & $1.062 \pm 0.196$ & $0.978 \pm 0.211$ & 0.182 \\
\hline
\end{tabular}

Abbreviations: HbA1c; glycated hemoglobin A (HbA1c), FBS; Fasting blood sugar (mg/dL), 2HPP; 2-hours postprandial plasma glucose (mg/dL).

significant reductions in FBS level and angina frequency in the ranolazine group at 4- and 8-week post-intervention. Nevertheless, no significant alternations were seen in these parameters in the control group $(P=0.134)$. On the other hand, the duration of the exercise test $(P=0.353)$ and serum creatinine $(P=0.182)$ were similar in both groups. 

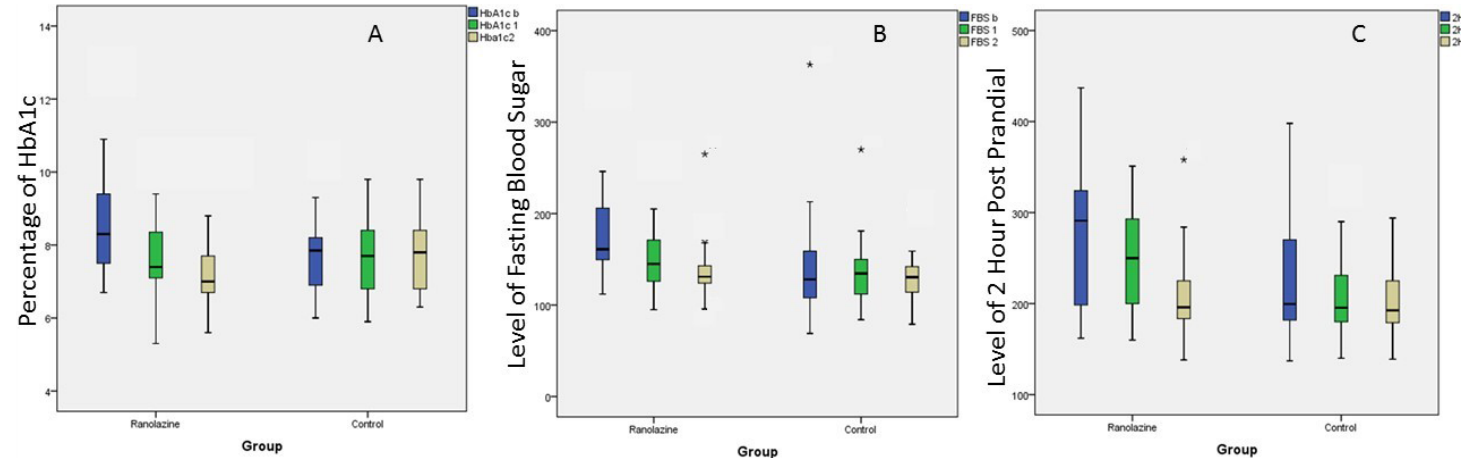

Figure 2. Alternations of $\mathrm{Hb} \mathrm{A1c}$, FBS, and $2 \mathrm{HPP}$ levels in the studied groups. (A) HbA1c percentage reduced in ranolazine treated group over the time while no significant change was observed in the conventional therapy group during the 8 weeks of study $(P=0.072)$. (B) FBS level reduced in both groups during the study with more pronounced reduction in the ranolazine group $(P=0.011)$. (C) The $2 \mathrm{HPP}$ levels showed a significant reduction in both ranolazine and control groups compared to the baseline $(P=0.011)$.

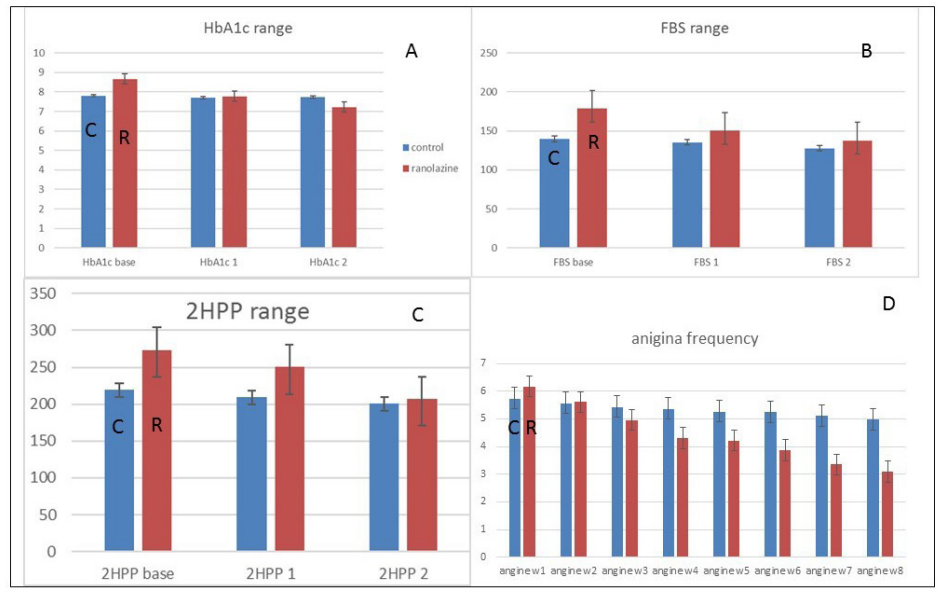

Figure 3. The changes in $\mathrm{Hb} \mathrm{A1c}$, FBS, 2HPP, and angina frequency in the studied groups. A) HbA1c significantly reduced in ranolazine following 8 weeks of treatment. B) FBS level significantly reduced in ranolazine group. C) While a decreasing trend was seen in the level of $2 \mathrm{HPP}$ in the ranolazine group, there was no significant difference comparing the mean difference of $2 \mathrm{HPP}$ between ranolazine and control groups after two months of intervention $(P=0.56)$. D) the number of angina attacks per week significantly reduced in both ranolazine and conventional therapy groups.

This indicates that ranolazine therapy can decrease HbA1c level in patients with concurrent T2DM and angina treated with conventional anti-diabetes therapies without increasing the risk of hypoglycemia. Nevertheless, there is insufficient data to conclusively conclude that ranolazine does not exaggerate the risk of hypoglycemia.

DM is a risk factor of CAD and angina $(1,2)$. Although there have been significant improvements in the management of heart diseases, the burden of mortality and morbidity due to heart disease still remains high (17). Recently, the beneficial roles of drugs modulating the energy metabolism and mitochondrial function of myocardial cells have been shown. These agents enhance the ability of myocardium to effectively metabolize glucose and to increase the overall ATP production (18, 20). Ranolazine, an approved antianginal drug, is capable of inhibiting cardiac late sodium currents (21). In patients with T2DM, however, ranolazine administration reduced HbAlc percentage $(3,22)$ which was in accordance with our study.

HbAlc measurement is the gold standard indicator of glycemic status and is widely used as a diagnostic and prognostic marker in diabetes (23). In addition, increased $\mathrm{HbA1c}$ is considered an independent risk factor for cardiovascular diseases. Nevertheless, HbA1c level is influenced by fluctuations of hemoglobin, administration of medications, and the ethnicity of patients. Another limitation of $\mathrm{HbAlc}$ is that, it can only reflect the mean blood glucose level during the recent two to three months. Furthermore, HbA1c measurement cannot accurately reflect short-term glycemic variability in patients. Finally, $\mathrm{HbA1c}$ alternations are delayed limiting its application for monitoring the therapeutic effects of glucose-reducing agents. 


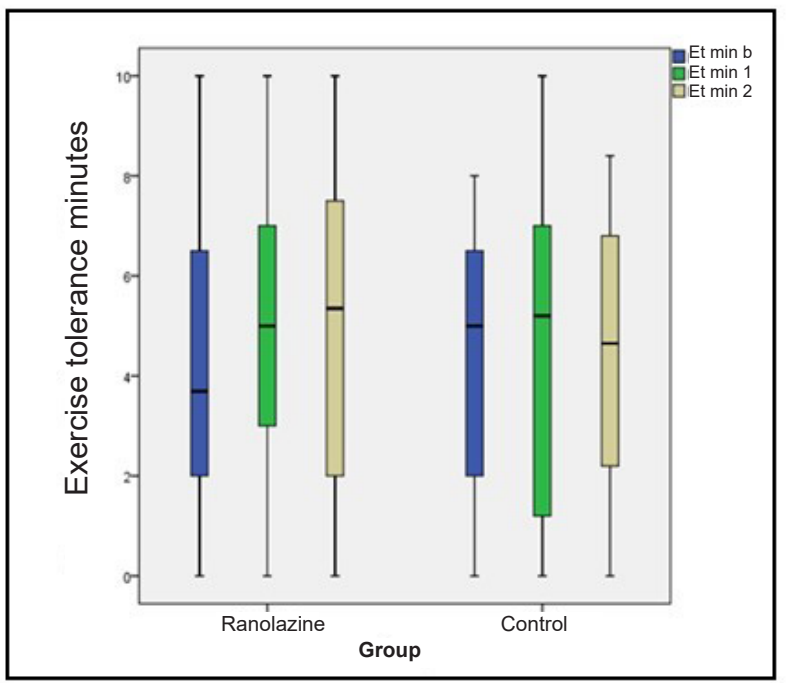

Figure 4. The exercise tolerance test in both studied groups at the baseline, and after 4 and 8 weeks of intervention. No significant change was observed in the exercise tolerance duration comparing the two groups $(P=0.353)$.

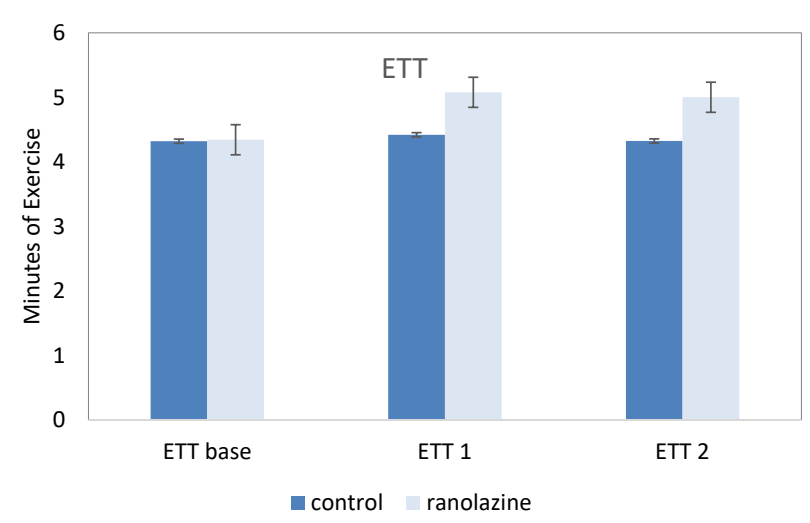

Figure 5. The exercise tolerance test (ETT) in both studied groups. Ranolazine significantly increased the capability of patients to perform the exercise test while no improvement was observed in patients that received conventional therapy.

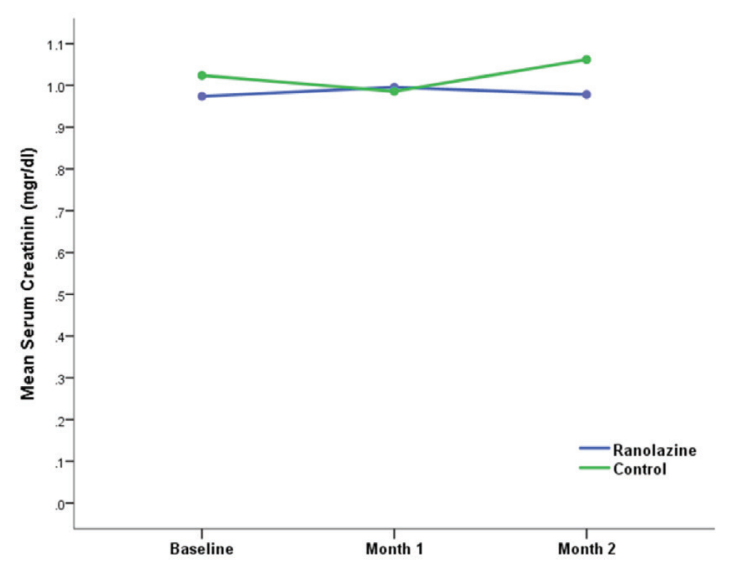

Figure 6. Serum creatinine levels at baseline and after 4 and 8 weeks of intervention.
Table 3. The incidence of adverse events $(\mathrm{AE})$ in diabetic patients with angina received either ranolazine or conventional therapy

\begin{tabular}{|c|c|c|}
\hline \multirow{2}{*}{ Adverse event } & \multicolumn{2}{|c|}{ Diabetic patients with angina } \\
\hline & Control group ${ }^{a}$ & Intervention group ${ }^{b}$ \\
\hline Patients with $\mathrm{AE}$ & 6 & 8 \\
\hline Discontinuation due to $A E$ & 0 & 0 \\
\hline Constipation & 2 & 4 \\
\hline Dizziness & 1 & 1 \\
\hline Nausea & 1 & 1 \\
\hline Asthenia & 2 & 2 \\
\hline Hypoglycemia & 0 & 0 \\
\hline Other Adverse Events & 0 & 0 \\
\hline
\end{tabular}

a $500 \mathrm{mg}$ metformin TDS, $80 \mathrm{mg}$ gliclazide BID, $2.6 \mathrm{mg}$ nitroglycerin daily. b $500 \mathrm{mg}$ metformin TDS, $80 \mathrm{mg}$ gliclazide BID, $1000 \mathrm{mg}$ ranolazine BID.

Ranolazine is an antianginal drug with cardioprotective features without any undesirable impacts on cardiac hemodynamic features such as heart rate and blood pressure. Ranolazine has been effective in the treatment of chronic angina both as monotherapy and in combination with commonly prescribed cardiovascular drugs $(24,25)$. The type 2 diabetes mellitus evaluation of ranolazine in subjects with chronic stable angina (TERISA) clinical trial was the first study to highlight the efficiency of ranolazine in reducing the frequency of angina episodes in diabetic patients who had high baseline HbA1c levels (8). Similarly, other studies have revealed the anti-hyperglycemic effects of ranolazine either alone or in combination with other agents $(5,26,27)$. However, more studies are required to determine the optimal ranolazine-based pharmaceutical regimens to augment its therapeutic effects. Our patients who received ranolazine had improved exercise tolerance time which is in line with previous studies expressing that ranolazine significantly improved exercise duration compared with placebo $(3,28)$. We also demonstrated that higher doses of ranolazine and longer durations of treatment can dose-dependently enhance the therapeutic effects of the drug in reducing HbAlc and FBS levels.

One of our objectives was to ascertain whether ranolazine could reduce the frequency of angina attacks in our patients. In this regard, the patients in both groups experienced chest pain every day at the beginning of the study. After receiving ranolazine; however, the patients in the intervention group mentioned a significant reduction in chest pain while patients in the control group expressed no alternation. According to our findings, it can be estimated that longer periods of ranolazine therapy (for instance 12 weeks) may result in disappearance of angina attacks in diabetic patients.

\section{Conclusion}

Ranolazine treatment can reduce the occurrence of angina attacks and improve quality of life and exercise tolerance 
time in patients with concurrent T2DM and chronic stable angina. In addition, ranolazine was shown as a welltolerated therapy reducing $\mathrm{HbAlc}$ level in these patients without causing renal dysfunction or imposing serious adverse events such as hypoglycemia.

\section{Limitations of the study}

The major limitation of our study was that elderly patients had difficulties to perform exercise test as most of them complained about physical limitations such as knee problems. Secondly, uneducated patients had difficulties in filling out their forms.

\section{Authors' contribution}

BFF and GG conducted the research. FF, ZG and SMA conducted the first edition. Accordingly, AZM and HB conducted the second edition and revised the manuscript. All authors read and signed the final manuscript.

\section{Conflicts of interest}

Authors declare no potential conflicts of interest. Ranolazine was provided to the patients free of any charge.

\section{Ethical considerations}

Ethical issues (including plagiarism, double publication) were completely observed by the authors. A part of this study was presented as an abstract in ISCOMS; the International Student Congress of (bio)Medical Sciences (www.iscoms.com).

\section{Funding/Support}

The Simin Daroo Company sponsored the study.

\section{References}

1. Goraya TY, Leibson CL, Palumbo PJ, Weston SA, Killian JM, Pfeifer EA, et al. Coronary atherosclerosis in diabetes mellitus: a population-based autopsy study. J Am Coll Cardiol. 2002;40:946-53.

2. Berry C, Tardif J-C, Bourassa MG. Coronary heart disease in patients with diabetes: part I: recent advances in prevention and noninvasive management. J Am Coll Cardiol. 2007; 49:631-42. doi: 10.1016/j.jacc.2006.09.046.

3. Timmis AD, Chaitman BR, Crager M. Effects of ranolazine on exercise tolerance and $\mathrm{HbAlc}$ in patients with chronic angina and diabetes. Eur Heart J. 2006;27:42-8. doi: 10.1093/eurheartj/ehi495.

4. Björntorp P. "Portal" adipose tissue as a generator of risk factors for cardiovascular disease and diabetes. Arteriosclerosis. 1990;10:493-6. doi: 10.1161/01. ATV.10.4.493.

5. Eckel RH, Henry RR, Yue P, Dhalla A, Wong P, Jochelson $\mathrm{P}$, et al. Effect of ranolazine monotherapy on glycemic control in subjects with type 2 diabetes. Diabetes Care. 2015;38:1189-96. doi: 10.2337/dc14-2629.

6. Pompilio G, Gennari M, Gambini E, Bassetti B, Capogrossi MC. Refractory Angina Pectoris: Focus on Cell Therapy. Atherosclerotic Cardiovascular Disease. InTech; 2011. doi:

\section{$10.5772 / 21655$}

7. Stone PH, Chaitman BR, Stocke K, Sano J, DeVault A, Koch GG. The anti-ischemic mechanism of action of ranolazine in stable ischemic heart disease. J Am Coll Cardiol. 2010;56:934-42. doi: 10.1016/j.jacc.2010.04.042.

8. Kosiborod M, Arnold SV, Spertus JA, McGuire DK, Li Y, Yue $\mathrm{P}$, et al. Evaluation of ranolazine in patients with type 2 diabetes mellitus and chronic stable angina: results from the TERISA randomized clinical trial (type 2 diabetes evaluation of ranolazine in subjects with chronic stable angina). J Am Coll Cardiol. 2013;61:2038-45. doi: 10.1016/j. jacc.2013.02.011.

9. Coppini R, Ferrantini C, Yao L, Fan P, Del Lungo M, Stillitano $\mathrm{F}$, et al. Late sodium current inhibition reverses electro-mechanical dysfunction in human hypertrophic cardiomyopathy. Circulation. 2013;127:575-84. doi: 10.1161/CIRCULATIONAHA.112.134932.

10. Sossalla S, Maier LS. Role of ranolazine in angina, heart failure, arrhythmias, and diabetes. Pharmacol Ther. 2012;133:311-23. doi: 10.1016/j.pharmthera.2011.11.003.

11. Patel PD, Arora RR. Utility of ranolazine in chronic stable angina patients. Vasc Health Risk Manag. 2008;4:819-824.

12. Belardinelli L, Shryock J, Fraser H. Inhibition of the late sodium current as a potential cardioprotective principle: effects of the late sodium current inhibitor ranolazine. Heart. 2006;92:iv6-14. doi: 10.1136/hrt.2005.078790.

13. Nathan DM, Singer DE, Hurxthal K, Goodson JD. The clinical information value of the glycosylated hemoglobin assay. N Engl J Med. 1984;310:341-6. doi: 10.1056/ NEJM198402093100602.

14. Rohlfing CL, Wiedmeyer H-M, Little RR, England JD, Tennill A, Goldstein DE. Defining the relationship between plasma glucose and HbAlc: analysis of glucose profiles and $\mathrm{HbAlc}$ in the Diabetes Control and Complications Trial. Diabetes Care. 2002;25:275-8. doi: 10.2337/ diacare.25.2.275.

15. Blackburn B, Belardinelli L, Wolff A. Use of ranolazine for the treatment of non-coronary microvascular diseases. Google Patents. 2008.

16. Dargie HJ, Hildebrandt PR, Riegger GA, McMurray JJ, McMorn SO, Roberts JN, et al. A randomized, placebocontrolled trial assessing the effects of rosiglitazone on echocardiographic function and cardiac status in type 2 diabetic patients with New York Heart Association Functional Class I or II Heart Failure. J Am Coll Cardiol. 2007;49:1696-704. doi: 10.1016/j.jacc.2006.10.077.

17. Vasan RS, Wilson P. Epidemiology and causes of heart failure. UpToDate Online; 2007. Available from: https:// www.uptodate.com/contents/epidemiology-and-causes-ofheart-failure.

18. Fragasso G, Salerno A, Spoladore R, Bassanelli G, Arioli F, Margonato A. Metabolic therapy of heart failure. Curr Pharm Des. 2008;14:2582-91.

19. Doenst T, Nguyen TD, Abel ED. Cardiac metabolism in heart failure: implications beyond ATP production. Circ Res. 2013;113:709-24. doi: 10.1161/CIRCRESAHA.113.300376.

20. Loudon BL, Noordali H, Gollop ND, Frenneaux MP, Madhani M. Present and future pharmacotherapeutic agents in heart failure: an evolving paradigm. $\mathrm{Br} \mathrm{J}$ Pharmacol. 2016;173:1911-24. doi: 10.1111/bph.13480. 
21. Steggall A, Mordi IR, Lang CC. Targeting metabolic modulation and mitochondrial dysfunction in the treatment of heart failure. Diseases. 2017;5:14.

22. Morrow DA, Scirica BM, Chaitman BR, McGuire DK, Murphy SA, Karwatowska-Prokopczuk E, et al. Evaluation of the glycometabolic effects of ranolazine in patients with and without diabetes mellitus in the MERLIN-TIMI 36 randomized controlled trial. Circulation. 2009;119:2032-9. doi: 10.1161/CIRCULATIONAHA.107.763912.

23. Sato A. Indicators of glycemic control--hemoglobin A1c (HbAlc), glycated albumin (GA), and 1, 5-anhydroglucitol (1, 5-AG). Rinsho Byori. 2014; 62:45-52.

24. Kloner RA, Hines ME, Geunes-Boyer S. Efficacy and safety of ranolazine in patients with chronic stable angina. Postgrad Med.2013;125:43-52.doi: 10.3810/pgm.2013.11.2711.
25. Tamargo J, Amorós I, Barana A, Caballero R, Delpón E. New investigational drugs for the management of acute heart failure syndromes. Curr Med Chem. 2010;17:363-90.

26. Page II RL, Ghushchyan V, Read RA, Hartsfield CL, Koch II BR, Nair KV. Comparative effectiveness of ranolazine versus traditional therapies in chronic stable angina pectoris and concomitant diabetes mellitus and impact on health care resource utilization and cardiac interventions. Am J Cardiol. 2015;116:1321-8. doi: 10.1016/j.amjcard.2015.08.002.

27. Lamendola P, Nerla R, Pitocco D, Villano A, Scavone G, Stazi $A$, et al. Effect of ranolazine on arterial endothelial function in patients with type 2 diabetes mellitus. Atherosclerosis. 2013;226:157-60.

28. Nash DT, Nash SD. Ranolazine for chronic stable angina. Lancet. 2008;372:1335-41.

Copyright $\odot 2020$ The Author(s); Published by Nickan Research Institute. This is an open-access article distributed under the terms of the Creative Commons Attribution License (http://creativecommons.org/licenses/by/4.0), which permits unrestricted use, distribution, and reproduction in any medium, provided the original work is properly cited. 